\title{
Personalized Service Recommendation Based on Trust Relationship
}

\author{
Hao Tian ${ }^{1,2}$ and Peifeng Liang ${ }^{2}$ \\ ${ }^{1}$ School of Information Engineering, Hubei University of Economics, No. 8, Yangqiaohu Ave., Jiangxia Dist., Wuhan 430205, China \\ ${ }^{2}$ Graduate School of Information, Production and Systems, Waseda University, Hibikino 2-7, Wakamatsu-ku, \\ Kitakyushu 808-0135, Japan
}

Correspondence should be addressed to Hao Tian; haotian@whu.edu.cn

Received 19 November 2016; Revised 4 February 2017; Accepted 14 February 2017; Published 7 March 2017

Academic Editor: Hiromitsu Shimakawa

Copyright (C) 2017 Hao Tian and Peifeng Liang. This is an open access article distributed under the Creative Commons Attribution License, which permits unrestricted use, distribution, and reproduction in any medium, provided the original work is properly cited.

\begin{abstract}
With the rapid development and extensive application of Web services, various approaches for Web service recommendation have been proposed in the past. However, the traditional methods only utilize the information of the user-service rating matrix but ignore the trust relations between users, so their recommendation precision is often unsatisfactory, and, furthermore, most of these methods lack the ability to distinguish the credibility of recommendation. To address the problems, we proposed a personalized service recommendation based on trust relationship. In particular, our approach takes into account user experience, interest background, recommendation effect, and evaluation tendency in the formalization of trust relationship, and moreover it can filter out useless or suspected services by exploiting trust relationships between users. To verify the proposed approach, we conducted experiments by using a real-world Web services set. The experimental results show that our proposed approach leads to a substantial increase in the precision and the credibility of service recommendations.
\end{abstract}

\section{Introduction}

With the rapid development of service computing, more and more Web services are published to the Internet. When a user submits a request, the system may return a large number of available Web services with the same function properties but with different nonfunction properties. It is difficult to select a high-quality Web service among these candidates. Therefore, recommending an appropriate service from a lot of Web services to fulfill a user's requirement is a nontrivial task.

Current service recommendation methods are usually based on collaborative filtering (CF) which is a successful and important technology in the research and application of recommender systems. The basic idea of CF is to predict and recommend potential favorite items for a particular user by employing rating data collected from other users [1]. However, since these methods treat each user without distinction, although the efficiency is high, due to a lack of personalization treatment, the precision of recommendations tends to be low. And it is worth noting that most of these approaches often give results under the assumption that each user recommends each service absolutely reliably, while ignoring the credibility of service recommendation. What is more, coldstart and sparsity still seriously devalue the performances of these methods. Thus, how to resolve the typical problems to enhance the quality of recommendations is becoming a research hotspot in the study of service recommendation.

In this paper, we formalize trust relationships between users by quantitating several factors which influence recommendation results and propose a service recommendation approach to enhance the precision and the credibility of recommendations, which can realize personalized recommendation based on trust relationship. The experimental results show that the method can effectively enhance the quality of recommendation.

This paper is organized as follows. In Section 2 we will review the related service recommendation methods as well as analyze their advantages and shortcomings. The quantitative methods for trust factors and the formalization for trust relationship are both introduced in Section 3. In 
Section 4 we elaborate on our proposed algorithm, followed by the experimental evaluations in Section 5. The conclusion is given in Section 6 .

\section{Related Work}

Service recommendation has been extensively studied in recent years. Based on QoS performances of Web services, various approaches have been proposed. Zheng et al. presented a collaborative filtering approach for predicting QoS values of Web services and making Web service recommendation by taking advantages of past usage experiences of service users [2]. Aiming to address the prediction of unknown QoS property values and the evaluation of overall QoS, a QoS evaluation for Web service recommendation is designed in [3]. In order to improve the recommendation accuracy, Chen et al. proposed an approach employing the characteristic of QoS, and they also used a recommendation visualization technique to show the result [4]. A Web services recommendation model based on QoS-awareness was proposed in [5]. The model could train Support Vector Machine (SVM) to gain a service selection function based on QoS information, and it could get a service candidate set by using the SVM classification method. In [6], the authors proposed an improved time-aware collaborative filtering approach for high-quality Web service recommendation. The approach integrated time information into both similarity measurement and QoS prediction. Although these approaches are generally feasible and efficient, because the QoS values of Web services are changing from time to time in reality, the applicability of this kind of recommendation methods is not satisfactory.

Besides the above methods, other approaches such as service recommendation based on users' location $[7,8]$, service reputation $[9,10]$, and context $[11,12]$ have been also proposed. Among them, with the advent of social networks, the trust-based approach to recommendation has emerged. In [13], the authors studied the trust relationships between users and built Web services using network. Based on the findings and the service network model, they then proposed a trust-based collaborative filtering algorithm to provide personalized service recommendations. Jamali and Ester explored a model-based approach for recommendation in social networks by employing matrix factorization techniques [14]. Advancing previous work, they incorporated the mechanism of trust propagation into the model. In order to counter malicious attack from recommending users, Wang et al. employed a beta trust model for the establishment of trust relationship between users [15]. Based on the combination of improved similarity calculation method and the trust degree of service recommendation behavior, a trustworthy community was set up to find neighbor. Similarly, a method for Web services recommendation based on social network trust relationship was designed in [16]. The algorithm utilizes the direct trust values and indirect trust values of services. Specifically, it recommends the services with direct trust values based on users' experiment and could recommend services based on indirect trust values if there are no experiences between the social network nodes and services. Generally, since these methods specially generate every recommendation by utilizing trust relationship between users, they can achieve better precision than the ones based on QoS.

In brief, traditional service recommendation methods have limitation in accuracy and applicability due to the timevariability of QoS values of Web services, and, furthermore, they hardly have the ability to distinguish the credibility of recommendation. On the other hand, the methods based on trust show the potentiality to address the shortcomings. In our previous research $[17,18]$, a user personalized model and a network model based on users' community relations are proposed. The user personalized model is constituted by two submodels: implicit preferences model and explicit preferences model. The implicit preferences model, based on domain ontology, can be used to explore a user's potential interest and reflect the user's preference continuity; the explicit preferences model, based on the user's preference ontology, can be used to express the user's nonfunctional requirements and reflect the real-time user's preference. In the network model, community relations between users are decomposed into clustering relations, recommended relations, and trust relations. The formalization methods of the three relations are discussed and given, including the relevant quantification and calculation methods. Consequently, on the basis of this work, we design a personalized Web service recommendation based on trust relationship, which can effectively formalize and use the trust relationship between users, to improve the precision and credibility of recommendation and, thus, to realize personalized recommendation.

\section{Formalization of Trust Relationship}

We argue that the trust relationship between users should be based on user experience, and it may be characterized by the level of trust, which mainly depends on the similarity of user's interest background, each recommendation effect, and evaluation tendencies of users. Therefore, the quantitative methods for these factors are given as follows.

3.1. User Experience. In our view, a user always recommends a Web service according to the satisfaction after invoking the service; in other words, user experience can be denoted by the evaluation value about services. Hence, for a user $u_{n}$, his/her user experience is a matrix $\mathbf{E}_{\mathbf{n}}$, which can be described as

$$
\begin{aligned}
& \mathbf{E}_{\mathbf{n}}=\left[\begin{array}{cccc}
e_{11}^{n} & e_{12}^{n} & \cdots & e_{1 k}^{n} \\
e_{21}^{n} & e_{22}^{n} & \cdots & e_{2 k}^{n} \\
\vdots & \vdots & \ddots & \vdots \\
e_{i 1}^{n} & e_{i 2}^{n} & \cdots & e_{i k}^{n}
\end{array}\right] \\
& e_{i k}^{n}=f\left(s_{i}, q_{k}\right), s_{i} \in \mathbf{S}_{\mathbf{n}}, q_{k} \in \mathbf{Q},
\end{aligned}
$$

where matrix $\mathbf{S}_{\mathbf{n}}$ is the services set invoked by $u_{n}$ and matrix $\mathbf{Q}$ expresses the set of QoS parameters. According to the definitions by W3C [19], the QoS requirements for Web services mainly refer to the quality aspect of a Web service. These may include performance, reliability, scalability, 


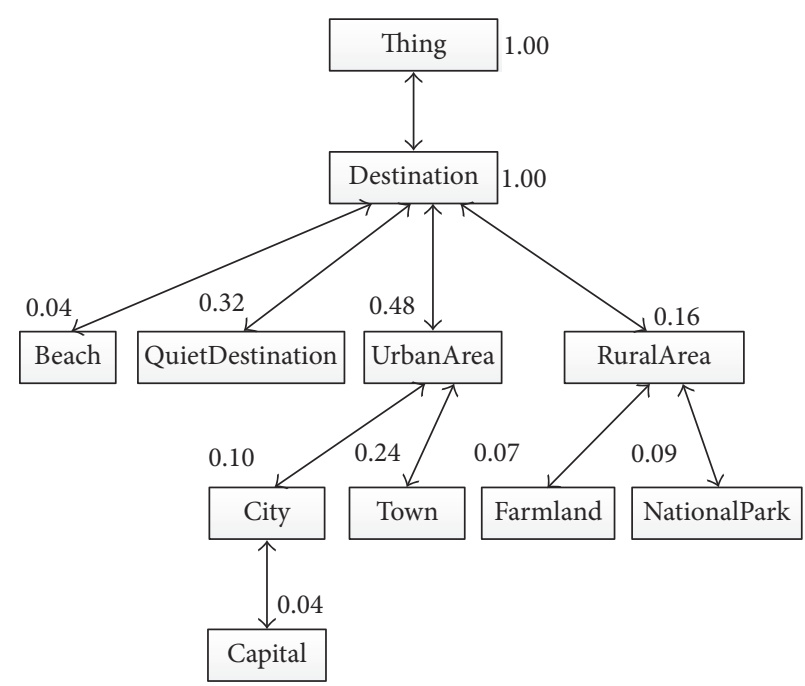

(a) User preference models of $u_{a}$

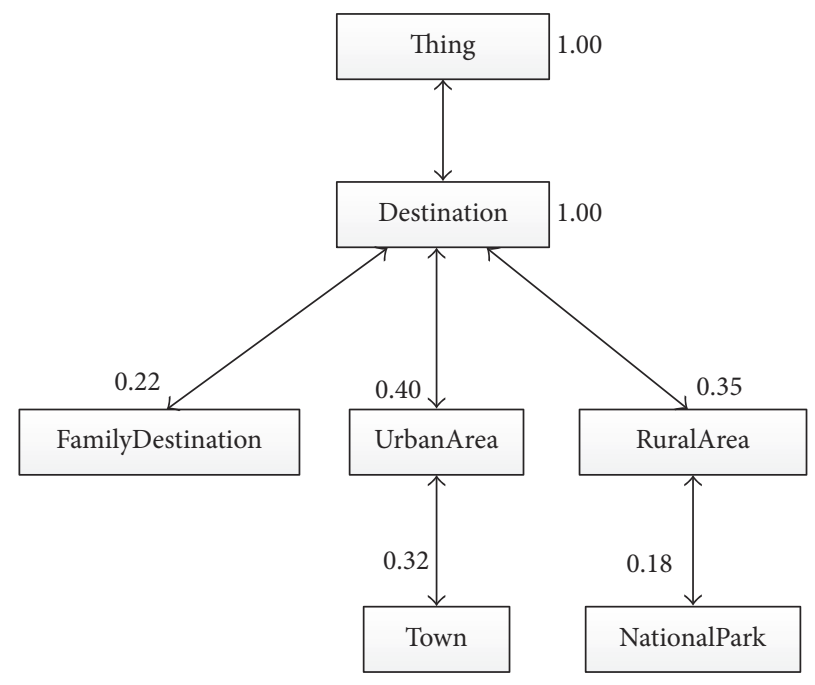

(b) User preference models of $u_{b}$

FIGURE 1: A sample of user preference models.

capacity, robustness, exception handling, response time, and throughput. In practical application, these factors usually can be monitored and detected after being invoked. In this paper, $q_{k}$ denotes the typical QoS factors such as price, response time, and throughput.

In traditional recommendation systems, the target service is usually evaluated by users with an overall satisfaction rating, and it is generally believed that the users giving the same rating values on the same services are similar users with same interest. However, there is a significant problem that these approaches cannot distinguish users' preferences of interest or evaluation tendencies. To address such problems, we set the evaluated object as a combination of several QoS parameters.

In the above matrix, $e_{i k}^{n}$ denotes the rating value of service $s_{i}$ on QoS parameter $q_{k}$ by user $u_{n}$. Particularly, $e_{i k}^{n}$ can be any real number, but often ratings are integers in the range $[1,5]$. In this paper, we take the same measure.

3.2. Interest Background. This paper argues that the more similar the users' interest backgrounds, the higher the credibility of recommendation; that is, user's interest background is a prerequisite to build a trust relationship. Therefore, discussion about the effect of users' interest backgrounds on mutual trust relationship is actually a comparison between their interest backgrounds. For a user set $\mathbf{U}=\left\{u_{1}, u_{2}, \ldots, u_{n}\right\}$, the similarities between interest backgrounds are given in a matrix B:

$$
\mathbf{B}=\left[\begin{array}{cccc}
b_{11} & b_{12} & \cdots & b_{1 n} \\
b_{21} & b_{22} & \cdots & b_{2 n} \\
\vdots & \vdots & \ddots & \vdots \\
b_{n 1} & b_{n 2} & \cdots & b_{n n}
\end{array}\right]
$$

We suggest that similarity between interest backgrounds depends on the similarity between user preference models, namely, a user's preference model can denote his/her interest background. In a previous study [17], we have proposed a user preference model based on ontology and designed the similarity calculating method between models, so we adopt them in this paper. Specifically, for $u_{a}, u_{b} \in \mathbf{U}, b_{a b} \in \mathbf{B}$ is the similarity between them, $0 \leq b_{a b} \leq 1, b_{a a}=1$, and $b_{a b}=b_{b a}$.

Figure 1 shows a sample of user preference models which are essentially subtrees from domain ontology. Since the structure of domain ontology can cover almost all standard concepts and categories of the domain, hence, for a specific user, by analyzing and refining his/her historical operations record and call records, it is feasible to reveal the interesting points which can perform as a local node of domain ontology.

As mentioned above, in this example, the user preference models in Figure 1 actually, respectively, denote the interest backgrounds of $u_{a}$ and $u_{b}$. By utilizing the pruning strategy proposed in [17], we can generate the matching tree between the user preference models of $u_{a}$ and the user preference models of $u_{b}$, which is shown as in Figure 2. Then, we can calculate the similarity between interest backgrounds of $u_{a}$ and $u_{b}$ based on the matching tree; namely, we can get the value of $b_{a b}$. In Figure $2, b_{a b}=0.712$.

3.3. Recommendation Effect. It is clear that a user's evaluation on a recommended service will impact on the degree of trust to the recommender, and the evaluation can be reflected by recommendation effect. In this paper, we use the difference between user experiences on the recommended services as the main indicator to measure the effect of each recommendation.

If user $u_{a}$ recommends a service $s_{i}$ to user $u_{b}$ and $u_{b}$ calls and evaluates $s_{i}$, then the recommendation effect from $u_{a}$ to $u_{b}$ on $s_{i}$ is $f_{a \rightarrow b}^{i}$, which can be calculated as follows:

$$
f_{a \rightarrow b}^{i}=\frac{\sum_{q_{k} \in \mathbf{Q}}\left(e_{i k}^{b}-e_{i k}^{a}\right)}{e_{\max } \times n_{q}},
$$




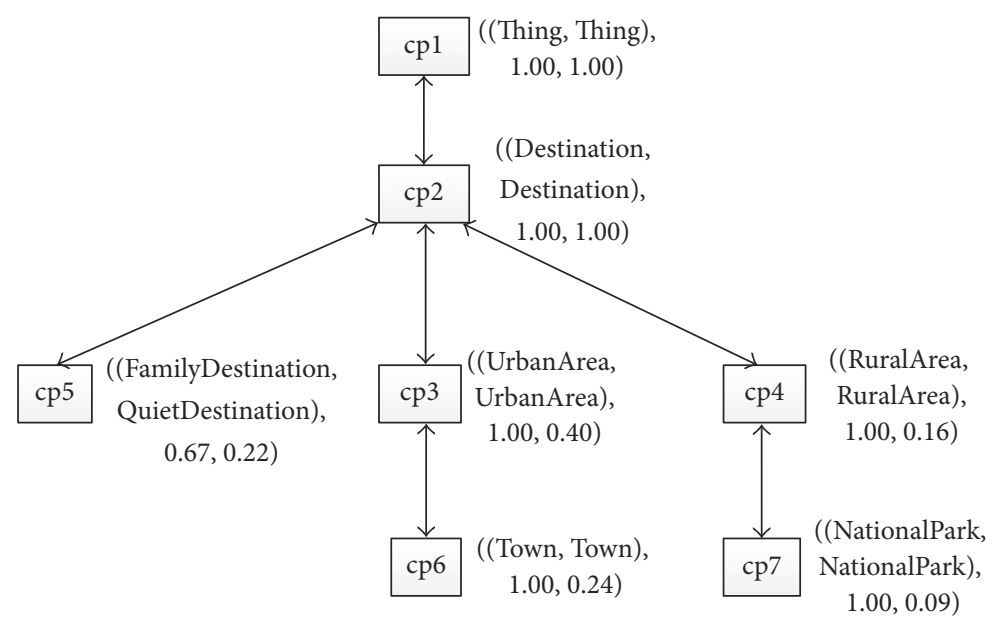

Figure 2: A sample of matching tree between user preference models.

where $e_{i k}^{a}$ and $e_{i k}^{b}$ are, respectively, the user experiences of $u_{a}$ and $u_{b} ; e_{\max }$ is the max rating value of service on QoS parameter in the range, as mentioned before, $e_{i k}^{n}$ is stated as integer in the range $[1,5]$, and, thus, $e_{\max }=5$ in this paper; $n_{q}$ is the number of QoS parameters in matrix $\mathbf{Q}$.

From this, it can be seen that $f_{a \rightarrow b}^{i} \neq f_{b \rightarrow a}^{i}$, and if $f_{a \rightarrow b}^{i}>$ 0 , this recommendation will increase the trust of $u_{b}$ to $u_{a}$; else if $f_{a \rightarrow b}^{i}=0$, the trust will be maintained in previous level; otherwise, the recommendation will damage the trust.

In this paper, we suppose that a server can record every action such as recommendation, calling, and evaluation, and the server can also maintain and update all trust relationships in real time. Moreover, we also assume that every action is complete and independent.

3.4. Evaluation Tendency. Tendency evaluation will also affect the trust relationship between users. Simply put, user's rating for different parameters will reflect their evaluation tendencies. Concrete saying: if two users give the same total scores for the same service but for some specific parameters the rating values are different, then you can believe that their evaluation tendencies are not the same. Namely, we can compare users' evaluation tendencies based on the rating values of these specific parameters.

In the user-based CF recommendation, the neighbors of each user are usually generated by calculating the similarity between users, and Pearson Correlation Coefficient [20] and Jaccard Similarity Coefficient [21] are the two typical methods. They can be described by the following equations:

$$
\begin{aligned}
& \operatorname{PCC}_{a b} \\
& =\frac{\left|\sum_{s_{i} \in \mathrm{S}_{\mathrm{ab}}}\left(\sum_{q_{k} \in \mathbf{Q}}\left(e_{i k}^{a}-\overline{e_{k}^{a}}\right) \times \sum_{q_{k} \in \mathbf{Q}}\left(e_{i k}^{b}-\overline{e_{k}^{b}}\right)\right)\right|}{\sqrt{\sum_{s_{i} \in \mathrm{S}_{\mathrm{ab}}} \sum_{q_{k} \in \mathbf{Q}}\left(e_{i k}^{a}-\overline{e_{k}^{a}}\right)^{2}} \times \sqrt{\sum_{s_{i} \in \mathrm{S}_{\mathrm{ab}}} \sum_{q_{k} \in \mathbf{Q}}\left(e_{i k}^{b}-\overline{e_{k}^{b}}\right)^{2}}} \\
& \mathrm{JSC}_{a b}=\frac{n_{a b}}{n_{a}+n_{b}-n_{a b}},
\end{aligned}
$$

where $n_{a}$ is the service number in $\mathbf{S}_{\mathbf{a}} ; n_{b}$ is the service number in $S_{b} ; S_{a b}$ is the matrix of services called and evaluated by both $u_{a}$ and $u_{b}$; and $n_{a b}$ is the service number in $\mathbf{S}_{\mathbf{a b}}$.

From (4) we can see that Pearson Correlation Coefficient can better take advantage of the user's scores, but the credibility of its conclusions is restricted by the number of recommended objects. On the other hand, Jaccard Similarity Coefficient can effectively resolve the problem of the number of objects in the former but failed to use the specific rating values, and its result is rough. Therefore, the effective combination of the two approaches is a worthy anticipated program. In this paper, we design a method to calculate the similarity between evaluation tendencies based on these two approaches, which is shown as

$$
\begin{aligned}
d_{a b}= & \mathrm{PCC}_{a b} \times \operatorname{JSC}_{a b} \\
& \times \frac{\sum_{s_{i} \in \mathrm{S}_{\mathrm{ab}}} \prod_{q_{k} \in \mathbf{Q}}\left(1-\left|e_{i k}^{b}-e_{i k}^{a}\right| / e_{\max }\right)}{n_{a b}},
\end{aligned}
$$

where $d_{a b}$ is the similarity between $u_{a}$ 's evaluation tendency and $u_{b}$ 's evaluation tendency; PCC $_{a b}$ and $\mathrm{JSC}_{a b}$ are the similarities calculated, respectively, by Pearson Correlation Coefficient and Jaccard Similarity Coefficient; $e_{\max }$ is the max rating value which has been defined in (3).

3.5. Trust Relationship. Based on the above factors, we can formalize the trust relationship between users. First, we should quantitate it by the degree of trust. By prior analysis we can conclude that the degree of trust between users is related to their interest backgrounds and evaluation tendencies and affected by the effect of each recommendation, which can be calculated as follows:

$$
t_{a b}=b_{a b} \times\left(1+\sum_{s_{i} \in S_{b \rightarrow a}} d_{a b} \times f_{b \rightarrow a}^{i}\right),
$$

where $t_{a b}$ is the degree of trust of $u_{a}$ to $u_{b}$, it represents the strength of trust relationship, and the greater its value, the 


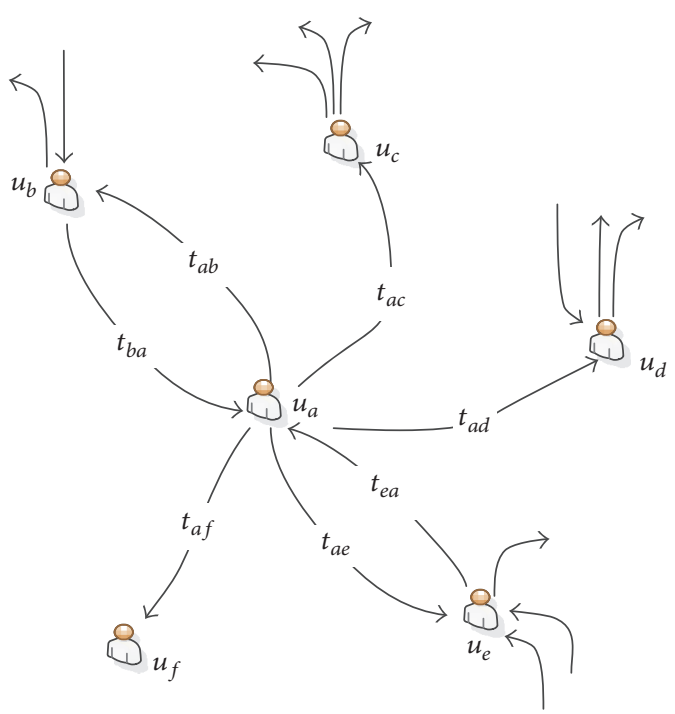

FIgURe 3: A trust relation sample.
Input: target user $u_{a}$

Output: recommended set $\mathbf{R}_{\mathbf{a}}$ of services for $u_{a}$

(1) Establish the trust relationship of $u_{a}$, namely, $T_{a}$

(2) $\mathbf{R}_{\mathbf{a}}=\phi$

(3) for each user $u_{m} \in \mathbf{U}_{a}$ do

(4) if $t_{a m}>t_{0}$ then

(5) for each service $s_{i} \in \mathbf{S}_{m}$ do

(6) calculate $p_{i}^{a}$

(7) if $p_{i}^{a}>p_{0}$ then

(8) add $s_{i}$ into $\mathbf{R}_{\mathrm{a}}$

(9) end if

(10) end for

(11) end if

(12) end for

(13) rank $\mathbf{R}_{\mathbf{a}}$ by $p_{i}^{a}$ in descending order

(14) return $R_{a}$ stronger the relationship; $S_{b \rightarrow a}$ is the set of services which $u_{b}$ recommended to $u_{a}$. According to this equation, it is clear that $t_{a b}$ will dynamically change with each recommendation from $u_{b}$ to $u_{a}$, and $t_{a b} \neq t_{b a}$.

Thus, a trust relationship is essentially a set of users and degrees of trust, which can be described in

$$
T_{a}=\left\{\left(u_{m}, t_{a m}\right) \mid u_{m} \in U_{a}, t_{a m}>0\right\},
$$

where $T_{a}$ is the trust relationship of $u_{a}$ and $U_{a}$ is the set of users in whom $u_{a}$ trusts. To be specific, once $u_{m}$ recommends a service to $u_{a}$ and $t_{a m}>0$, the trust from $u_{a}$ to $u_{m}$ will be established and $u_{m}$ then will be an element of $U_{a}$. Figure 3 shows a trust relation sample, in which there are five users in $U_{a}$. It should be noted that if $t_{a m} \leq 0$, the trust from $u_{a}$ to $u_{m}$ will be removed.

\section{Service Recommendation Based on Trust Relationship}

After building a trust relationship, a group of trusted users can be selected out. Based on this trust relationship, the target user's acceptances of services can be predicted and then some services can be recommended.

In this paper, the predicted acceptances of services can be calculated by

$$
p_{i}^{a}=t_{a b} \times \frac{\sum_{q_{k} \in \mathbf{Q}} e_{i k}^{b}}{\max _{s_{j} \in \mathbf{S}_{b}}\left(\sum_{q_{k} \in \mathbf{Q}} e_{j k}^{b}\right)},
$$

where $p_{i}^{a}$ indicates the predicted acceptance of service $s_{i}$ by $\operatorname{target}$ user $u_{a} ; \mathbf{S}_{\mathbf{b}}$ is the matrix of services invoked by $u_{b}$. Since we calculate recommendation effect, evaluation tendency, and user interest background similarity based on individual ratings to each QoS parameter in this paper, the degree of trust between users such as $t_{a m}$ in the above equation can effectively ensure the accuracy of prediction. Thus, in order to reduce the amount of computation, the above equation predicts only the overall rating of the target user to services on all QoS factors.

Based on the above work, a personalized service recommendation based on trust relationship (PSRTR) is proposed, and its specific steps are described in Algorithm 1.

In Algorithm 1, in order to enhance the relevance between recommended services and target user and reduce invalid calculations, we set two thresholds: $t_{0}$ and $p_{0}$; by utilizing them, we can also limit the number of services in $\mathbf{R}_{\mathbf{a}}$.

In traditional methods, it is natural to recommend highly evaluated services with an integrated predicted acceptance value over all users who evaluated a service. However, these highly evaluated services often cannot meet the target user's demand. Hence, to improve the precision of recommendation, the proposed method uses only individual predicted value based on trust relationship. It can not only enhance the pertinence of recommendation, but also ensure the reliability of recommendation.

It also can be seen that the final recommended services all have been called by the trusted neighbors of target user, and they are well evaluated in these neighbors' user experiences. Based on the trust relationship, the algorithm can avoid useless or suspected services, to improve the precision and credibility of recommendation.

\section{Experiment and Analysis}

In order to verify the performance of the method proposed in this paper, we performed the following simulation experiments in this section. Under the same experimental conditions, we compared it with several representative methods and analyzed the differences.

5.1. Experimental Setup. In this paper, we use a real-world QoS dataset from WS-DREAM [22, 23] which can monitor 339 Web services by using 5825 distributed computer nodes located all over the world. The dataset contains more than 
TABLE 1: Main characteristics of the WS-DREAM dataset.

\begin{tabular}{lc}
\hline Parameters & Values \\
\hline Number of users & 339 \\
Number of services & 5825 \\
QoS values & Response time and throughput \\
\hline
\end{tabular}

1,900,000 invoking records. Table 1 shows the main characteristics of it.

For comparison, we set the response time and throughput for the two QoS parameters involved in the evaluation. Based on this dataset, we adopt the method proposed in [24] to generate users' ratings for each Web service. Through utilizing these ratings, we get each user's preference model and then calculate the similarities between interest backgrounds by comparing the implicit preference models of user preference models based on the method presented in our previous study [17].

In the simulation, the user will be divided into two groups, namely, the training and test sets, of which $80 \%$ of users are assigned into the training set and the remaining $20 \%$ into the test set, and the users of the training set recommend services to the users of the test set according to the proposed method PSRTR.

The popular evaluation metric in recommendation is Mean Absolute Error (MAE) which can be defined as follows:

$$
\operatorname{MAE}=\frac{\sum_{i=1}^{N}\left(\left|e_{i}^{a}-e_{i}^{b}\right| /\left(n_{q} \times e_{\max }\right)\right)}{N},
$$

where $e_{i}^{a}$ is the actual overall evaluation value about service $s_{i}$ by user $u_{a} ; e_{i}^{b}$ is the overall user experience of $u_{b}$ about service $s_{i}$, namely, the predicted value, and these two factors can be generated based on the user experiences of $u_{a}$ and $u_{b}$ by the method in [24]; $N$ is the number of recommended services; $e_{\max }$ and $n_{q}$ have been defined in (3). Obviously, the smaller the value of MAE is, the higher the accuracy of the results recommended is.

In order to estimate the fitness of recommendation, namely, to verify how correctly the proposed method can predict the target user's QoS evaluation, we define Mean Subitem Absolute Error (MSAE) as another evaluation metric in our experiments, which can be described as follows:

$$
\text { MSAE }=\frac{\sum_{i=1}^{N} \sum_{q_{k} \in \mathbf{Q}}\left(\left|e_{i k}^{a}-e_{i k}^{b}\right| /\left(n_{q} \times e_{\max }\right)\right)}{N},
$$

where the definitions of $N, e_{i k}^{a}, e_{i k}^{b}, e_{\max }$, and $n_{q}$ are same as the ones mentioned before. Evidently the smaller the value of MSAE is, the higher the fitness of the recommendation is.

To evaluate the performance of our method we consider two comparison partners: Personalized QoS Prediction (PQP) [25] and Collaborative Filtering Recommendation Based on Double Neighbor Choosing (CF-DNC) [26]. PQP makes similarity mining and predicts QoS for Web services from consumers' experiences. The method is based on the assumption that the consumers, who have similar historical experiences on some services, would have similar experiences on other services. CF-DNC chooses the preferences

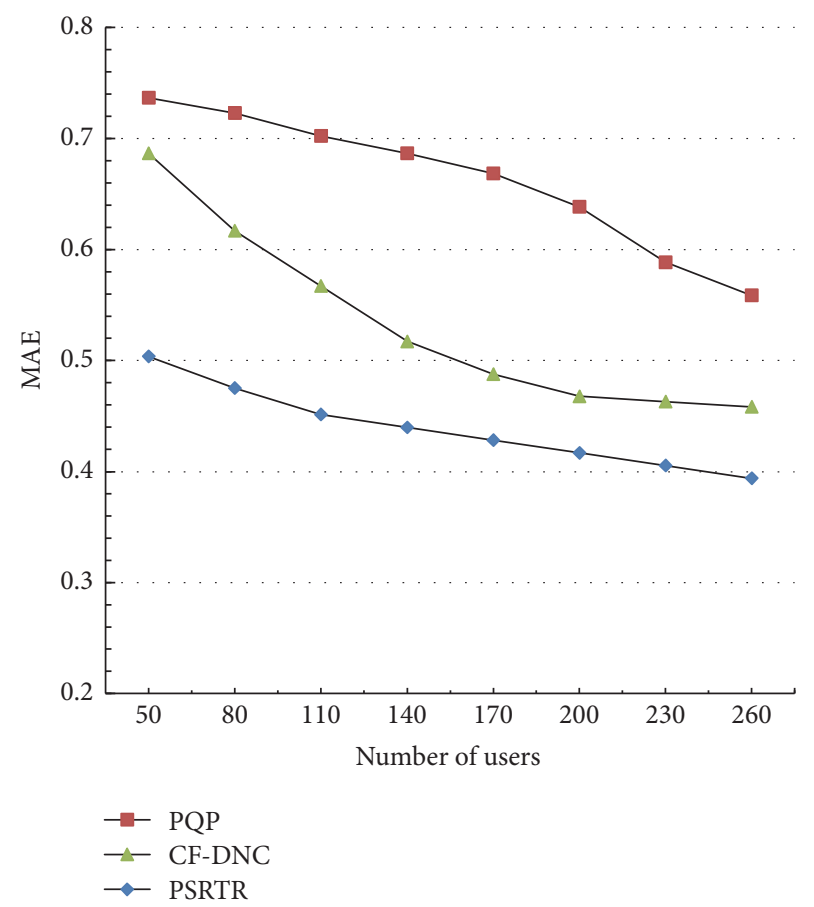

FIgURE 4: Comparison of precision.

similar users of target user dynamically on the basis of the computational result of user similarity and measures the trust relationship between users according to the ratings of similar users. These two approaches are partly similar to our proposed method on the principles of algorithms, so we selected them as the comparison partners in our experiments.

5.2. Results and Analysis. In the experiments, we set $t_{0}=0.6$, $p_{0}=0.3$, and we first select 50 users from WS-DREAM randomly and then increase users in steps of 30 until the number of total users reaches 260 , as mentioned earlier; $80 \%$ of them are included in the training set and the rest in the test set. Along with the increase of users, the changes about MAE and MSAE of these three methods are, respectively, shown in Figures 4 and 5.

As can be seen from Figure 4, the precision of PSRTR is far superior to the other two, which shows that once trust relationship is introduced into the recommendation process, we can improve the quality of recommendation result. Specifically, compared with the traditional collaborative filtering recommendation algorithm $\mathrm{PQP}$, the precision of the result of PSRTR is improved by $33.61 \%$ on average, and compared with the neighbor choosing algorithm CF-DNC, the precision is increased by $16.82 \%$ on average. Since the recommenders and the target users are highly similar in the interest backgrounds and evaluation tendencies, the results of PSRTR are more consistent with the preferences of the target users. Therefore, PSRTR can achieve the most satisfactory precision among these three algorithms.

Figure 5 shows the comparison result of the fitness of recommendation. It is clear that the fitness of PSRTR is also much better than the other two. In particular, the fitness of 


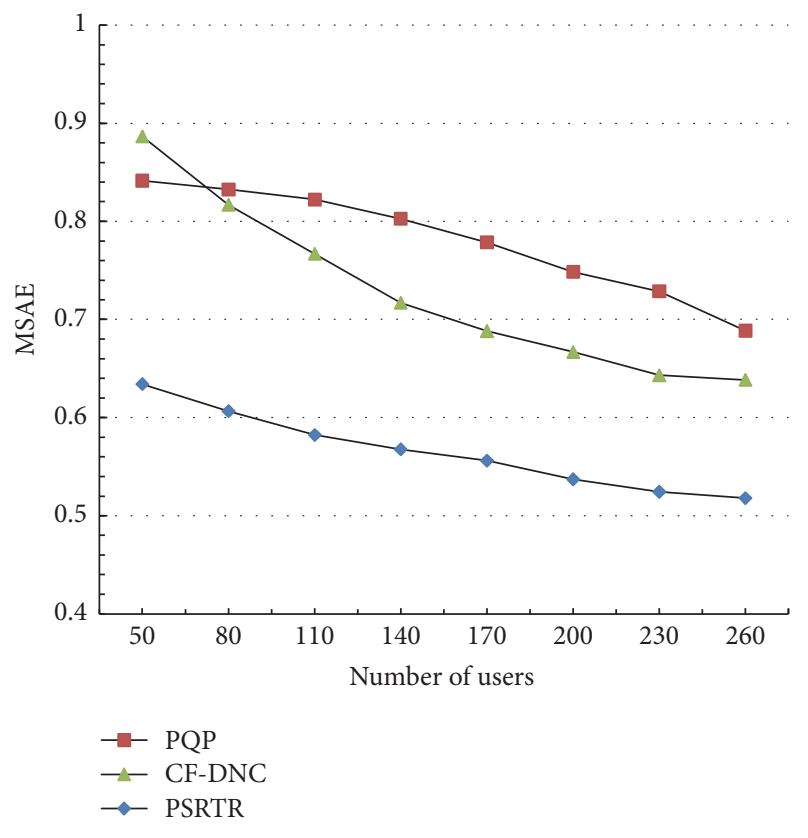

FIGURE 5: Comparison of fitness.

PSRTR is always the best from beginning to end. Compared with PQP, the MSAE of the result of PSRTR is improved by $37.93 \%$ on average, and compared with CF-DNC, the MSAE of the result of PSRTR is increased by $28.67 \%$ on average. Thus, it can be concluded that PSRTR can predict the target user's QoS evaluation most correctly among these three algorithms.

In order to validate the credibility of the above three methods, in this article, by gradually increasing the number of suspect users until $5 \%$ of the total users in each experiment, to verify their ability to withstand malicious recommendations, the experimental results are shown in Figure 6.

In Figure 6, we can see that when the number of suspect users in the system gradually increased, due to the lack of defense mechanism, the service recommendation precision of PQP method decreased rapidly; in contrast, the CFDNC method considers the issue of trust and has a certain antiattack capability; its precision declined relatively slowly. However, since the trust in the CF-DNC method is only based on the similarity of interests, as compared with the PSRTR method, its accuracy of similar users judging is not high, so its precision will be deteriorated with the increase of suspect users. On the contrary, the trust relationship in the PSRTR is built by a number of factors; once a trusted user generates malicious recommendation, the level of the trust of target user to him will be weakened, and the overall precision will not change much under the attacking from a small number of suspect users. Thus, it can be concluded that the PSRTR can well resist malicious recommendation, so its result has high credibility.

\section{Conclusion}

In this paper, we proposed a novel method to provide personalized Web service recommendations. Different from

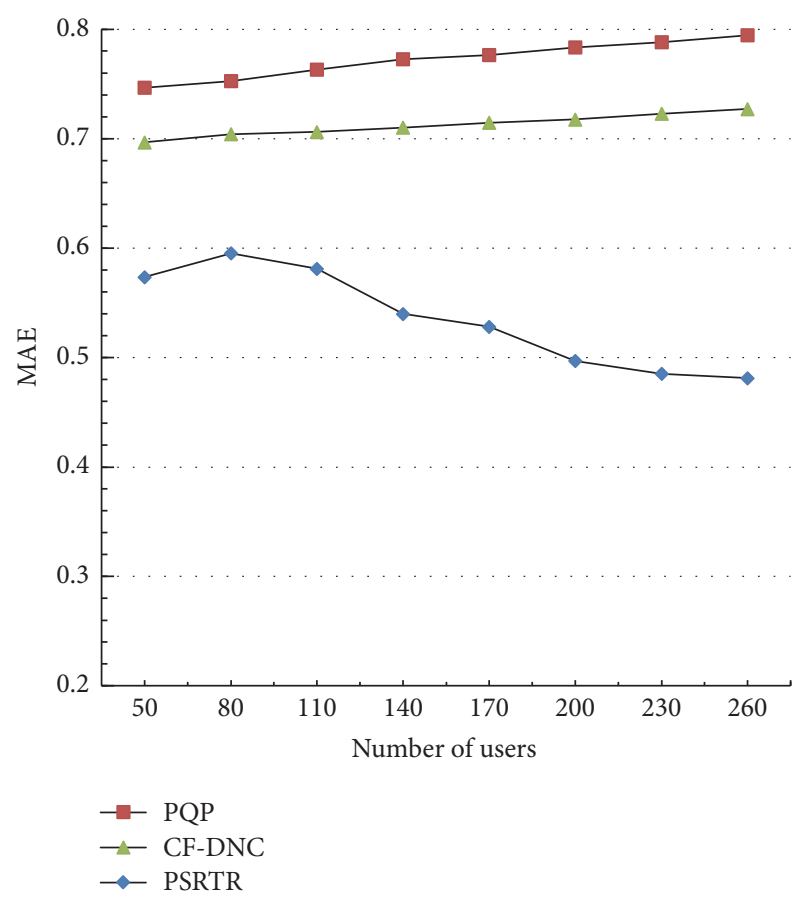

FIGURE 6: Comparison of credibility.

previous work, our algorithm constructs the trust relationship based on user experience and characterizes it by the level of trust. By quantitating the factors such as user's interest background, recommendation effect, and evaluation tendency of user, we realized the formalization of trust relationship. Based on trust relationship, we proposed a personalized service recommendation method named PSRTR. Experimental results show that our approach significantly improves the precision and credibility of recommendation compared with the existing methods. Future improvements that need to be addressed are how to further improve the algorithm performance, as well as how to consider other qualitative factors for trust relationship to further enhance the credibility of recommendation.

\section{Competing Interests}

The authors declare that there is no conflict of interests regarding the publication of this paper.

\section{Acknowledgments}

This work is supported by the Natural Science Foundation of Hubei Province, China, under Grant no. 2016CKB714.

\section{References}

[1] X. Chen, Z. Zheng, Q. Yu, and M. R. Lyu, "Web service recommendation via exploiting location and QoS information," IEEE Transactions on Parallel and Distributed Systems, vol. 25, no. 7, pp. 1913-1924, 2014. 
[2] Z. Zheng, H. Ma, M. R. Lyu, and I. King, "QoS-aware web service recommendation by collaborative filtering," IEEE Transactions on Services Computing, vol. 4, no. 2, pp. 140-152, 2011.

[3] M. You, X. Xin, W. Shangguang, L. Jinglin, S. Qibo, and Y. Fangchun, "QoS evaluation for web service recommendation," China Communications, vol. 12, no. 4, pp. 151-160, 2015.

[4] X. Chen, Z. Zheng, X. Liu, Z. Huang, and H. Sun, "Personalized QoS-aware web service recommendation and visualization," IEEE Transactions on Services Computing, vol. 6, no. 1, pp. 35-47, 2013.

[5] X. Jiang, Y. Wei, and B. Qiu, "QoS-aware web services personalized recommendation," Computer Technology and Development, vol. 25, no. 12, pp. 85-90, 2015.

[6] Y. Hu, Q. Peng, X. Hu, and R. Yang, "Time aware and data sparsity tolerant web service recommendation based on improved collaborative filtering," IEEE Transactions on Services Computing, vol. 8, no. 5, pp. 782-794, 2015.

[7] S.-D. Liu and X.-W. Meng, "Approach to network services recommendation based on mobile users' location," Journal of Software, vol. 25, no. 11, pp. 2556-2574, 2014.

[8] B. Berjani and T. Strufe, "A recommendation system for spots in location-based online social networks," in Proceedings of the 4th Workshop on Social Network Systems (SNS '11), Salzburg, Austria, April 2011.

[9] K. Tserpes, F. Aisopos, D. Kyriazis, and T. Varvarigou, "A recommender mechanism for service selection in serviceoriented environments," Future Generation Computer Systems, vol. 28, no. 8, pp. 1285-1294, 2012.

[10] S. Nepal, Z. Malik, and A. Bouguettaya, "Reputation management for composite services in service-oriented systems," International Journal of Web Services Research, vol. 8, no. 2, pp. 29-52, 2011.

[11] M. Sohn, S. Jeong, J. Kim, and H. J. Lee, "Augmented contextbased recommendation service framework using knowledge over the Linked Open Data cloud," Pervasive and Mobile Computing, vol. 24, pp. 166-178, 2015.

[12] L. Liu, F. Lecue, N. Mehandjiev, and L. Xu, "Using context similarity for service recommendation," in Proceedings of the 4th IEEE International Conference on Semantic Computing (ICSC '10), pp. 277-284, IEEE, Pittsburgh, Pa, USA, September 2010.

[13] S. Deng, L. Huang, and G. Xu, "Social network-based service recommendation with trust enhancement," Expert Systems with Applications, vol. 41, no. 18, pp. 8075-8084, 2014.

[14] M. Jamali and M. Ester, "A matrix factorization technique with trust propagation for recommendation in social networks," in Proceedings of the 4th ACM Recommender Systems Conference (RecSys '10), pp. 135-142, Barcelona, Spain, September 2010.

[15] H.-Y. Wang, W.-B. Yang, S.-C. Wang, and S.-R. Li, "A service recommendation method based on trustworthy community," Chinese Journal of Computers, vol. 37, no. 2, pp. 301-311, 2014.

[16] P. Zhang, B. Huang, R. Xie, and C. Chen, "A method for web services recommendation based on social network trust relationship," Journal of Chinese Computer Systems, vol. 35, no. 2, pp. 222-227, 2014.

[17] H. Tian, Research on User-Centered Web Service Discovery and Its Application in Financial Services, Wuhan University, Wuhan, China, 2014.

[18] H. Tian, H. Fan, and W. Du, "Research on Web service discovery based on user community relations," Journal on Communications, vol. 36, no. 10, pp. 28-36, 2015.
[19] QoS for Web services: requirements and possible approaches, 2015, http://www.w3c.or.kr/kr-office/TR/2003/ws-qos/.

[20] U. Shardanand and P. Maes, "Social information filtering: algorithms for automating "word of mouth", in Proceedings of the SIGCHI Conference on Human Factors in Computing Systems, pp. 210-217, Denver, Colo, USA, May 1995.

[21] L. Candillier, F. Meyer, and F. Fessant, "Designing specific weighted similarity measures to improve collaborative filtering systems," in Proceedings of the 2008 IEEE International Conference on Data Mining, pp. 242-255, Pisa, Italy, 2008.

[22] Z. Zheng, Y. Zhang, and M. R. Lyu, "Distributed QoS evaluation for real-world Web services," in Proceedings of the IEEE 8th International Conference on Web Services (ICWS '10), pp. 83-90, IEEE, Miami, Fla, USA, July 2010.

[23] Y. Zhang, Z. Zheng, and M. R. Lyu, "Exploring latent features for memory-based QoS prediction in cloud computing," in Proceedings of the 30th IEEE International Symposium on Reliable Distributed Systems (SRDS '11), pp. 1-10, IEEE, Madrid, Spain, October 2011.

[24] S.-G. Deng, L.-T. Huang, J. Wu, and Z.-H. Wu, "Trust-based personalized service recommendation: a network perspective," Journal of Computer Science and Technology, vol. 29, no. 1, pp. 69-80, 2014.

[25] L. Shao, J. Zhang, Y. Wei, J. Zhao, B. Xie, and H. Mei, "Personalized QoS prediction for web services via collaborative filtering," in Proceedings of the IEEE International Conference on Web Services (ICWS '07), pp. 439-446, Salt Lake City, Utah, USA, July 2007.

[26] D. Jia and F. Zhang, "A collaborative filtering recommendation algorithm based on double neighbor choosing strategy," Journal of Computer Research and Development, vol. 50, no. 5, pp. 10761084, 2013. 

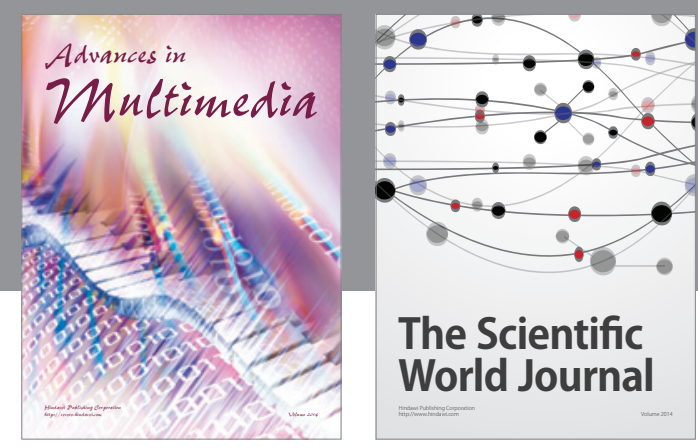

The Scientific World Journal
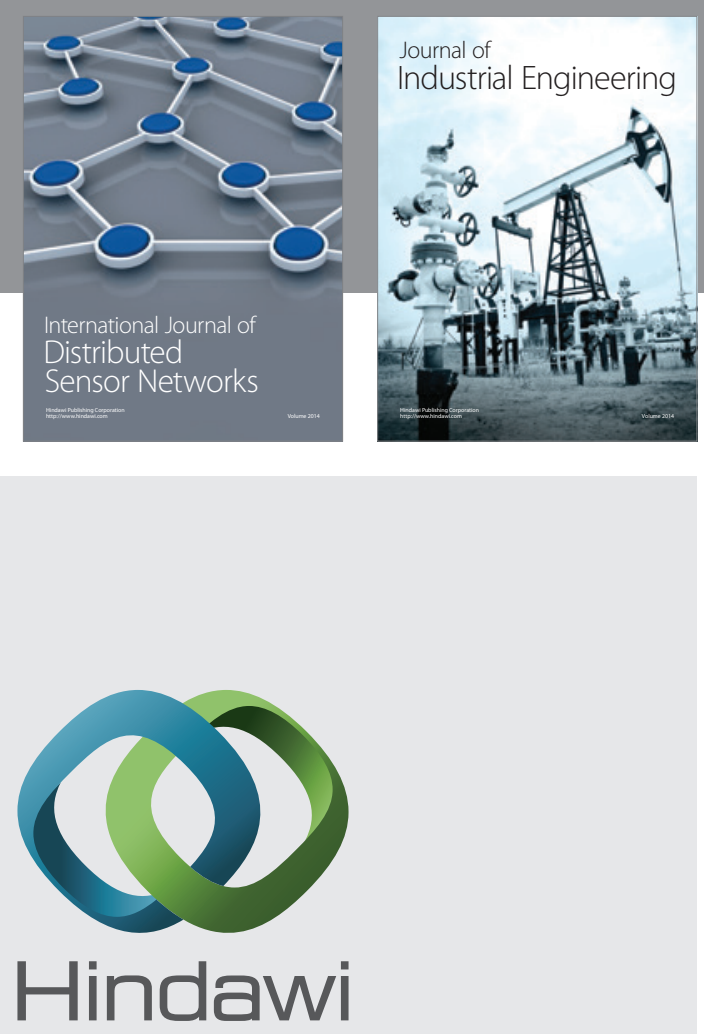

Submit your manuscripts at

https://www.hindawi.com

\section{Computer Networks} and Communications
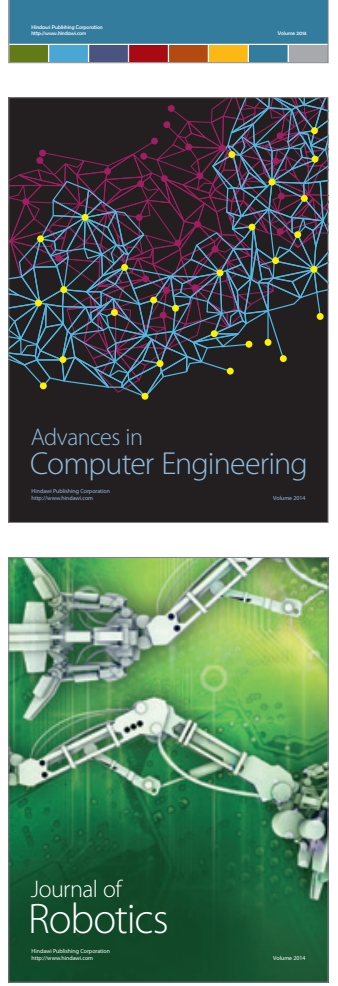
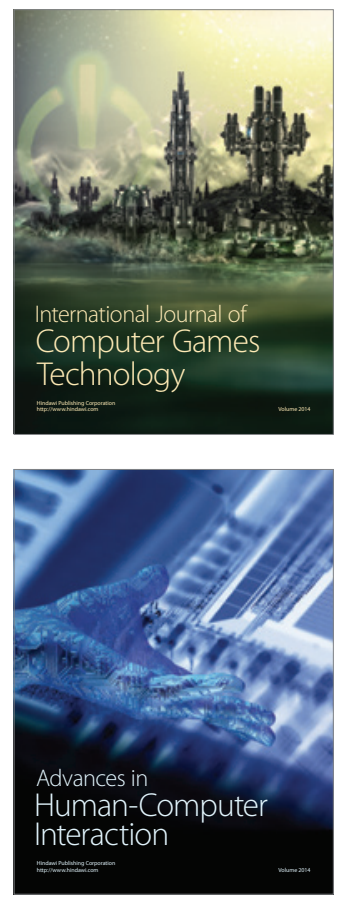
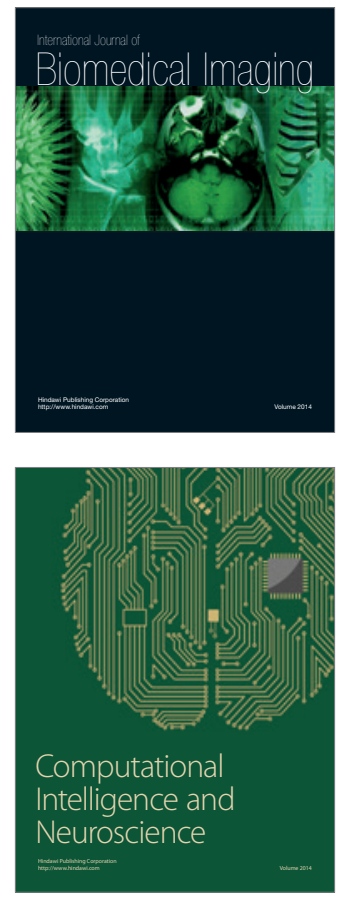
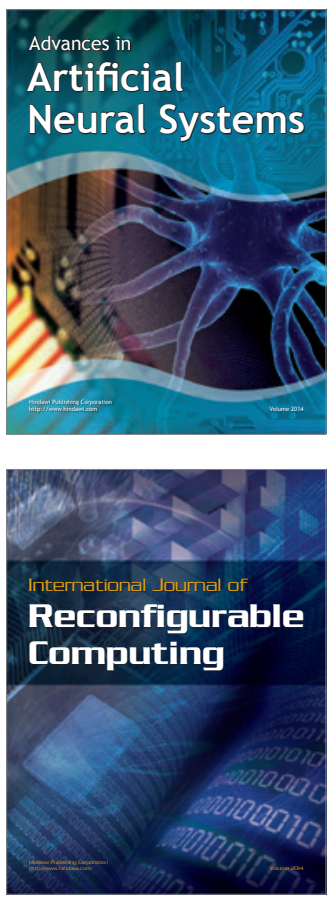
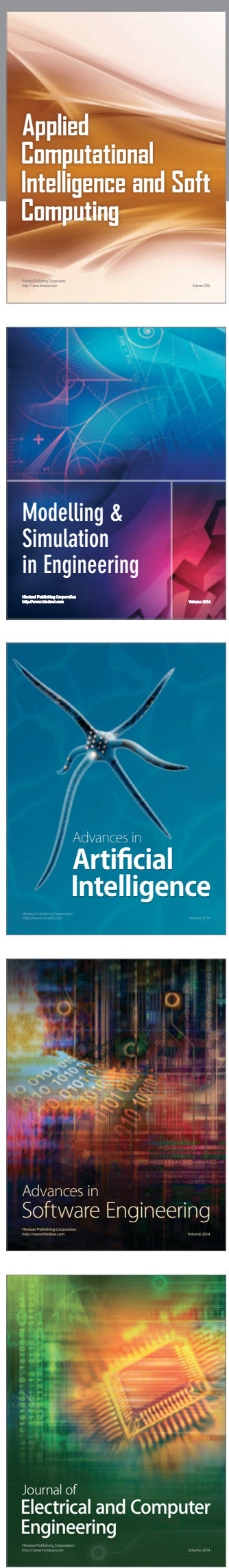